\title{
The Planet's Response to Human Activity. Thermodynamic Approach
}

\author{
Vladimir Kh. Dobruskin \\ Beer Yaakov, Israel \\ Email: dobruskn@gmail.com
}

How to cite this paper: Dobruskin, V.Kh. (2021) The Planet's Response to Human Activity. Thermodynamic Approach. Open Journal of Ecology, 11, 126-135. https://doi.org/10.4236/oje.2021.112011

Received: January 1, 2021

Accepted: February 6, 2021

Published: February 9, 2021

Copyright $\odot 2021$ by author(s) and Scientific Research Publishing Inc. This work is licensed under the Creative Commons Attribution International License (CC BY 4.0).

http://creativecommons.org/licenses/by/4.0/

cc) (i) Open Access

\begin{abstract}
The applicability of the laws of thermodynamics to processes on Earth is discussed and it is shown that the chemical thermodynamics provides the reasonable basis for predicting probable changes. The historical evolution of the planet is considered in the framework of the Harari approach; a civilization's level is estimated by the Kardashev scale based on the amount of energy it is able to use. During a short historical interval ( $\approx 500$ years), when the effect of biological evolution is imperceptible and the main changes on the planet are caused by human activity, two systems are considered: 1) a nonequilibrium inhabited planet and 2) a quasi-equilibrium hypothetical planet without people, which is accepted as a comparison system. It is shown that in response to the energy impact, the equilibrium of the hypothetical system with the primordial nature is disturbed and processes are initiated aimed to prevent further growth of energy production. In the case of a real planet, this implements changes preventing the uncontrolled activities of humans-the energy producers. Climate change, an increase in the number of natural disasters and epidemics can be recognized as a direct response of the planet, while changes in socio-economic relations, morality, demographic situation, new threats etc. can be considered as an indirect reaction to changing conditions of human beings. The latter results from the mutual correlation between the progress of society, on the one hand, and humanitarian and political processes, on the other. The role of renewable and non-renewable energy sources in evolution is taken into account. Obviously, it is better to take meaningful measures to achieve an acceptable balance now than to wait for the blind and extremely painful action of the laws of nature, which would lead to a reduction in the population.
\end{abstract}

\section{Keywords}

Planet's Response, Human Activity, Evolution 


\section{Introduction}

In recent decades, events in the world around us have been changing at breakneck speed. This applies to all aspects of life: the climate, the number of natural disasters, outbreaks of new diseases and the coronavirus pandemic, changes in morals and demographics, violent political struggles, conflicts of interests between the greens and industry, etc. The problems of the future of our civilization have been widely discussed in thousands of publications around the world, the full overview of which cannot be presented here. In most publications, consideration is based on the implicit assumption that development of events will proceed as usual and will not be any obstacles to progress. The very serious warning that it is not so was expressed by a group of scientists, which studied the possible limit of civilization growth [1] [2] [3]. These studies were initiated by the Club of Rome, which consisted of current and former heads of state and government, diplomats, scientists, economists, and business leaders from around the globe; the Club was created to address the multiple crises facing humanity and the planet. In the first report [1], the model used by the authors predicted resource depletion by 2072, which would lead to a sudden and uncontrolled decline in both population and industrial capacity. These studies generated debates around the world: some researchers supported the authors, but most sharply criticized them. In regard to this discussion, we adhere to the view of Avery, who stated that the basic thesis-that unlimited economic growth on a finite planet is impossible-was indisputably correct [4].

In contrast to the specific problem of resource depletion, we intend to discuss probable future changes in the framework of thermodynamics (TD), the foundations of which rest on the most general laws of physics. To do this, it is necessary 1) to substantiate the applicability of the laws of thermodynamics to events on the planet, 2) to discuss the impact of increasing energy consumption on environmental processes, and 3) to try to link changes in the global ecosystem of the Earth with the future development of human society. The author believes that the above-mentioned disturbing events in the world are connected with the planet's response to perturbating human activity.

\section{Earth as a Thermodynamic System. A Planet without People}

Any application of the laws of thermodynamics begins with an imaginary division of the Universe into a system (area of interest) and the rest-the environment. Since the universe is an isolated system, its entropy, $S$, can only grow:

$$
\Delta S \geq 0
$$

where equality is possible only if equilibrium would be reached- "the heat death of the universe". Consider our planet together with the atmosphere as a TD system and space as the environment; we will limit ourselves to events that occur during short time intervals compared to the period of the planet's existence. It should be emphasized that this is an important limitation, without which further 
consideration will be incorrect. The short interval in this case is the period of the last $\approx 500$ years of our history.

It is known that from space the main impact on our planet has solar radiation. A study of the earth's energy balance between incoming shortwave solar radiation, on the one hand, and outgoing radiation from the Earth, on the other, shows [5] that on average for a year energy inflow to the Earth's system, 341 $\mathrm{W} / \mathrm{m}^{2}$, is equal to the return radiation to space. Hence, on average the change in the Earth's thermal energy is zero, $q=0$. More detailed information about the energy balance can be found in the original source [5].

In addition, it is obvious that Earth and space practically do not exchange matter: a volume, $V$, and a mass of the planet remain practically unchanged. Although there has been an increase in temperature in recent years, the absolute increments are small compared to the average thermal temperature $T=288^{\circ} \mathrm{K}$ $\left(15^{\circ} \mathrm{C}\right)$. For example, over 138 years $(1880-2018)$ of observation [6], the temperature varied in the range from $-0.5^{\circ} \mathrm{C}$ to $+1^{\circ} \mathrm{C}$ from the average temperature of the twentieth century. Therefore, we can roughly assume that the processes during small intervals occur at a constant temperature. The planet does not perform any work, $A$, in space, and in the formulation of the first law of thermodynamics

$$
q=\Delta U+A
$$

where $\Delta U$ is the change of the system internal energy, all constituents on average are equal to zero.

The evolution of the Earth after the origin of life was actually determined by the both geological and biological evolutions of the planet. The relatively calm course of events was disrupted by catastrophes of cosmic origin, which led to sharp singularities on an imaginary smooth curve of property-time. We do not consider such discontinuities, limiting ourselves to smooth periods. Biological evolution, with the exception of protozoan evolution, is a slow process that requires thousands of years or more before significant changes occur. Therefore, in the absence of people, a hypothetical uninhabited planet can be considered on average as a quasi-equilibrium system with $q=0, V=$ const, and $T=$ const for, say, 500 years. This is a dynamic equilibrium when at the macro level, on average for a year and over the entire surface, changes are not visible, but at the "molecular" micro level, processes continue: winds blow, water evaporates, seasons change, rains come, organisms are born and die, etc. Once again note that it is the entire planet that is in equilibrium, and not its individual constituencies. For example, a living organism is an open system that exchanges matter and energy with the environment and is never in equilibrium with it. For a living organism, equilibrium is death. The question of what life is from the point of view of TD has been studied by Schrodinger [7].

\section{Applicability of the Laws of Thermodynamics}

Our task is to predict the reaction of the planet to human activity in the global ecosystem of the Earth, that is, in the biosphere. The latter is the shell of a planet, 
populated by living organisms, which is under their influence and occupied by the products of their vital activity. The biosphere can be viewed as a huge collection of extremely complex chemical processes. For phase transitions, individual and conjugate reactions, the TD methods allow to calculate equilibrium data, temperature dependences, pressure effects, theoretical yields, and so on, and predict the effect of changes in the environment on the equilibrium parameters. Chemical thermodynamic [8] is one of the main tools of physical chemistry. The results of such quantitative TD calculations are often expressed qualitatively in the form of the Le Chatelier principle: if an external influence is exerted on a system that is in a state of dynamic equilibrium, then processes will occur in the system aimed at reducing this external influence. If we use a metaphor, we can say that the Le Chatelier principle reflects the existence of feedback between the system and the environment.

For the totality of all the most complex processes in the biosphere, which are based on chemical reactions, it is impossible to make the same quantitative calculations as for individual and conjugate reactions. However, due to the applicability of TD to individual reactions, it can be assumed that it is also applicable to their totality, at least for qualitative predictions. It should be noted that classical thermodynamics was developed before the generally accepted molecular-kinetic theory and does not require knowledge of: 1) the details of the object structure (in contrast to statistical TD) and 2) the molecular mechanisms of processes in the system. In our case, this means that to apply the laws of classical TD to the planet, one does not need to know the mechanism of processes in the biosphere.

It is generally accepted to consider biological evolution within the framework of Darwin's theory. The latter assumes that only organisms with genetic mutations, which make it possible to exist in a new environment, survive during changes in the environment. Harari [9] points out that the smooth biological evolution ended with the emergence of Homo sapiens who, in the course of the cognitive revolution, learned to quickly correct their behavior and pass on new skills to the next generations-for this they no longer needed any genetic mutations or changes in the environment. From that moment, the historical evolution starts. This is the point of bifurcation when biological and historical evolution begins to diverge. By the term "historical evolution", we mean the change in time of the totality of processes in human society and the environment, which are generated by the mental activity of mankind. It is obvious that both evolutions occur simultaneously, while people are subject to the same physical laws and cannot go beyond the limits established by biology. Unlike slow biological evolution (for example, sapiens have not changed physiologically even in tens of thousands of years of existence), historical processes are relatively fast, and changes can be noticeable during the life of one generation of people. Consequently, after the appearance of Homo sapiens, our planet is no longer in equilibrium even for short time intervals, but for about 500 years, we can: 1) ignore the contribution of biological evolution and 2) consider all changes as the result of historical evolution. In a populated non-equilibrium system with $V=$ const 
and $T=$ const, the processes will proceed in the direction of decreasing the isochoric-isothermal potential (Helmholtz potential), $F=U-T S$, [8].

The application of thermodynamics requires consideration of all components included in the energy balance (Equation (2)). In this regard, it is necessary to focus on the role of information. The cognitive revolution led to the development of outstanding abilities of Homo sapiens and the dominance of man in nature. Harari notes that we live in a world largely created by our imagination. Culture, ideology, morality, political parties, states, money, nations, stocks, laws, religion, science, human rights do not exist in nature and are examples of the manifestation of our cognitive abilities. A person's activity is completely determined by his mental activity. To characterize the latter, the concept of "information" is often used [10] [11] [12] and changes in the world are explained by the explosive growth of information. Historical evolution and accumulated information are in one-to-one correspondence. Both human activity and energy production, in particular, are based on information and are impossible without it. When we talk about the growth of information or the growth of energy production, it is equivalent to a discussion in different languages about the progress of civilization. Therefore, the use of information as a separate additional term in TD (energy) balance (Equation (2)) is incorrect and equivalent to double accounting for the same value.

\section{The Planet's Response}

In 1964, Kardashev proposed to assess the level of civilization's development by the amount of energy that it is able to use [13]. This is a generalized indicator of human activity in the language of physics: it covers all kinds of activity. Note the values of energy production and consumption are almost the same, since humanity does not accumulate any energy reserves for future. The Kardashev idea seems quite reasonable for assessing the development of human society; for example, the level of country's development is often compared by the amount of energy consumption per capita. The growth of energy consumption, $E$, began after the appearance of Homo sapiens about 50,000 years ago. For most of this period, the state of nature was little different from the world without peoples. About 500 years ago, the scientific and technological revolution began, which was based on a sharp, perhaps almost exponential increase in energy production. We are interested in this particular period of history. Consider two systems (Table 1):

1) Imagine a hypothetical world without peoples. For 500 years, it would have been in equilibrium with the environment. We will accept such a world as a reference system. Untouched by civilization, the primeval nature of this hypothetical world is the ideal for humanity.

2) The second system is our real inhabited and nonequilibrium world. The difference between the systems is related to human activity, expressed in the amount of energy produced (Figure 1). 


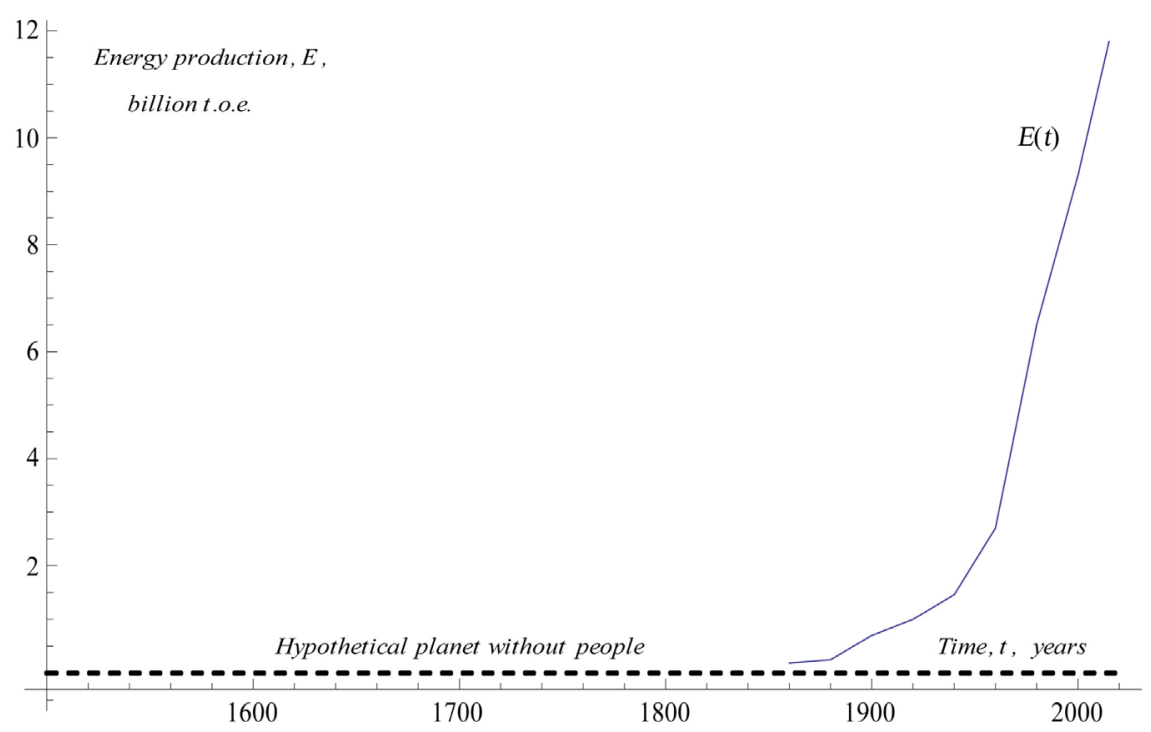

Figure 1. Energy production in billion tons of oil equivalent, (t.o.e) [14].

Table 1. Thermodynamical objects under consideration.

\begin{tabular}{|c|c|c|c|}
\hline $\begin{array}{l}\text { Objects under } \\
\text { consideration }\end{array}$ & System & Environment & $\begin{array}{l}\text { Object characteristics } \\
\text { and process direction }\end{array}$ \\
\hline Universe & Planet Earth & Space & $\begin{array}{l}\text { Nonequilibrium object. } \Delta S \geq 0 \text {. } \\
\text { Here } S \text { is the entropy of the Universe }\end{array}$ \\
\hline $\begin{array}{l}\text { Inhabited } \\
\text { planet Earth }\end{array}$ & Biosphere & $\begin{array}{l}\text { The rest of } \\
\text { the planet }\end{array}$ & $\begin{array}{l}\text { Biosphere is nonequilibrium object, } \\
\Delta F=\Delta U-T \Delta S \geq 0 . \text { Here } U \text { and } S \text { are } \\
\text { parameters of the system. }\end{array}$ \\
\hline $\begin{array}{l}\text { Hypothetical planet } \\
\text { Earth without people }\end{array}$ & $\begin{array}{l}\text { Earth } \\
\text { without people }\end{array}$ & Space & $\begin{array}{l}\text { Quasi-equilibrium object, } \\
q=0, V \text { and } T=\text { const. }\end{array}$ \\
\hline
\end{tabular}

Let's ask the question: "What would happen in a hypothetical equilibrium system if it is impacted by a destabilizing energy?" The answer to this question was considered earlier: if a system in a state of dynamic equilibrium is affected by an energy disturbance, then, in accordance with the Le Chatelier principle, processes are initiated in it aimed at countering this disturbance. An outer observer (for example, somebody from space) who evaluates the processes on the entire real planet and for whom it is seen as an aggregate of a hypothetical planet and energy producers will see that processes aimed at reducing the activity of sapiens are taking place on the entire planet. Namely it is the planet's response to the human activity. Naturally, the more $E$, the stronger the reaction. This conclusion relates only to the production of energy from non-renewable sources (NRS).

Let's consider this point in more detail. Renewable energy on the planet originates from the current energy of solar radiation. It does not depend on whether the planet is inhabited or not. In the case of an uninhabited planet, most of the solar energy is spent on maintaining the state of the planet, including life, some is converted into other forms of energy or stored in natural storage-deposits. 
In the case of our inhabited planet, a small fraction of it is used in wind-, solar-, tidal- and hydroelectric power plants to produce useful work for people, which in the process of use will also turn into heat. For example, an electric car carries a load, but all useful energy will be spent on overcoming friction and eventually also turns into heat. Therefore, renewable energy, which serves as the energy source of life, has the same impact on the planet without people and on the real planet. In the case of NRS, the situation is changing: one may say that it is stored, but not current, solar energy. The formation of NRS lasts for hundreds of millions of years, but the release of energy occurs very fast in the process of burning. Despite its partial use as useful work for people, the combustion energy will eventually also dissipate as heat, since there are no serious storage facilities for it. The general amount of combustion energy, i.e., the integral $Q=\int_{-50000}^{t} E(t) \mathrm{d} t$, rather than $E(t)$ has a destabilizing effect on the environment, where $t$ is time. One can roughly estimate the increase in the entropy of the planet due to the use of NRS from the appearance of Homo sapiens about 50,000 years ago to 2020:

$$
\Delta S=Q / T
$$

where $Q=\int_{-50000}^{2020} E \mathrm{~d} t$. Since there is no data for the entire period, we will limit ourselves to calculating $\Delta S$ from 1900 to 2020 years, based on the values shown in Figure 1. We get that for this period, human activity led to an increase in the entropy of the planet by $7.8 \times 10^{16} \mathrm{~kJ} \cdot \mathrm{K}^{-1}$ or per unit of land area by 524 $\mathrm{kJ} \cdot \mathrm{K}^{-1} \cdot \mathrm{m}^{-2}$. In accordance with the statistical meaning of the concept of "entropy", this value can be considered as a measure of increasing chaos, expressed in entropic units.

\section{Countering the Growth of Energy Consumption}

Even if we do not take into account the damage done to nature, historical evolution cannot continue indefinitely due to the depletion of resources and will inevitably begin to move back to a reduction in energy consumption and population [1] [2] [3]. In this paper, a general characteristic of a civilization is used-an energy consumption-and its impact on the conditions of existence in the environment is analyzed. Uncontrolled habitat destruction can occur before the most important non-renewable energy sources have been depleted. The value of the integral $Q=\int_{-50000}^{t} E(t) \mathrm{d} t$ (the area under the curve $E(t)$ ) increases with time even if an inflection occurs on the curve and $E$ begins to decrease. This means that the destabilizing effect will continue even after a decision to reduce $E$, i.e., there is a long-time interval between the decision and the achievement of a new quasi-equilibrium in the biosphere.

It is worth noting that the laws of nature do not directly affect energy production; but by changing the conditions of existence on the planet, they can regulate the number of energy producers. Of course, the most interesting question is: "How could the planet's response be realized?" One can reformulate this question: "How could the laws of nature force a decrease of the sapiens activity?" There are different possibilities for this, both direct and indirect. For example, in 
the arsenal of nature there are such formidable weapons as climate change and epidemics. A probability of the latter increases due to a growth in the rate of mutations of viruses in a contaminated environment. Another response can be traced in the chain of historical events. Harari believes [9] that historical evolution (moving forward) occurs due to the implementation of all the many manifestations of cognitive abilities in culture, ideology, morality, religion, social and economic relations, science, law, etc. As a result of thousands of years of development of these abilities and interaction between them, a modern society was formed. All the many manifestations of cognitive abilities are interconnected. From the above view of history and historical selection, it follows that the energy production, which characterizes the level of development of society, is associated with humanitarian and political processes, and this influence is mutual. For example, it is obvious that the situation when energy production corresponds to the 21st century, but the manifestation of cognitive abilities to the 16th century, is impossible. It can be assumed that in conditions of lack of resources and the constant clash of interests that form the society, the phenomena leading to a decrease in the population will begin to prevail. Here is an example of such a chain of events: the growth of energy consumption and well-being, access to education, advances in medicine, the emancipation of women, a decline in the birth rate, demographic changes and, as a result, a decrease in the population. Inevitably escalate the conflicts associated with the unequal distribution of civilization's achievements and struggle for resources. In practice, these are manifested in a sharp political struggle and emergence of new threats. All these questions are related to the field of politics, they are beyond the scope of this work.

It would like to note that there is a rather unusual hypothesis, the hypothesis of Lovelock [15], which has been rejected by the scientific community, but conclusions from the hypothesis are similar to those from the present study. Lovelock correctly suggested that from the moment of the origin of life, the planet should be considered as a single system consisting of the atmosphere, lithosphere, hydrosphere and biosphere, where each of its components affects the development of other components, and the evolution of life is closely related to the evolution of its physical environment on the scale of the entire Earth. But the Lovelock hypothesis, named after the ancient Greek Earth goddess "Gaia theory", wrongly claims that that the Earth and the global ecosystem of the planet behave as a biological superorganism, and not as an inanimate object. Those species of living beings that negatively affect the environment and make it less suitable for offspring will, Lovelock argued, eventually be expelled from the planet. From the very moment of the appearance of Lovelock's hypothesis, it was rightly criticized. Some of the fiercest opponents of the Gaia theory were Darwinian biologists [16]. They argued that the cooperation required for the self-regulation of the Earth could never be combined with the competition required for natural selection. Besides, the name taken from mythology also caused discontent, and the identification of the earth with superorganism con- 
tradicts the basic concepts of science. Although the theory was rejected, it does not prevent it from still exciting the imagination and gathering many supporters around the world.

\section{Conclusions}

The destructive consequences of human activity for the nature of the planet are well known. A thermodynamic examination of the planet's reaction shows that the laws of nature will prevent the sapiens' activity, which manifests itself in an uncontrolled increase in energy production, and such resistance will increase until a new equilibrium is established. The radical solution is to reduce energy production.

The most important question is: When will the planet reach the turning point, and the decline in population and energy production will become inevitable? It is clear that the turning point is not the extreme point on the geometric curve, but the time period when the processes, which negatively affect the environment, subside. It seems that the planet is close to the turning period and the evidence of this is increasing tensions in all areas that define human beings. Of course, there is no consensus on finding the critical point; the opinion expressed is individual and can be challenged. But the response resulting from thermodynamic consideration seems indisputable. Given what is at stake, it is hoped that the above conclusions will not be seen as another doomsday prophecy, and the sooner the better, meaningful measures will be taken to achieve a reasonable balance now, without waiting for the blind and extremely painful action of the laws of nature, which would lead to a reduction in the population.

\section{Conflicts of Interest}

The author declares no conflicts of interest regarding the publication of this paper.

\section{References}

[1] Meadows, D.H., Meadows, D.L., Randers, J.W., Behrens III, W.W. (1972) The Limits to Growth. Universe Books, New York.

[2] Meadows, D.H., Randers, J. and Meadows, D.L. (2004) The Limits to Growth: The 30-Year Update. Chelsea Green Publishing Co., Vermont.

[3] Randers, J. (2012) 2052: A Global Forecast for the Next Forty Years. Chelsea Green Publishing Co., Vermont.

[4] Avery, J.S. (2012) Information Theory and Evolution. World Scientific, Singapore. https://doi.org/10.1142/8441

[5] Kiehl, J.T. and Trenberth, K.E. (1997) Earth's Annual Global Mean Energy Budget. Bulletin of the American Meteorological Society, 78, 197-208. https://doi.org/10.1175/1520-0477(1997)078\%3C0197:EAGMEB\%3E2.0.CO;2

[6] Cole, S. (2019) Fourth Warmest Year in Continued Warming Trend, According to NASA, NOAA. The Earth Observer, 31, 24.

[7] Schrodinger, E. (1944) The Physical Aspect of the Living Cell. Cambridge Universi- 
ty Press, Cambridge.

[8] Atkins, P.W. (1980) Physical Chemistry. Izd. Mir, Moscow. (In Russian)

[9] Harari, Y.N. (2014) Sapiens: A Brief History of Humankind. Harvill Secker, London.

[10] Hartley, R.V.L. (1928) Transmission of Information. American Telephone and Telegraph Company, New York.

[11] Shannon, C.E. (1948) A Mathematical Theory of Communication. Bell System Technical Journal, 27, 379-423. https://doi.org/10.1002/j.1538-7305.1948.tb01338.x

[12] Volkenstein, M.V. (2009) Entropy and Information. Progress in Mathematical Physics, Vol. 57, Birkhäuser, Basel. https://doi.org/10.1007/978-3-0346-0078-1

[13] Kardashev, N.S. (1964) Transmission of Information by Extraterrestrial Civilizations. Soviet Astronomy, 8, 217-221.

[14] Stages of World Energy Development (2015) Institute for Energy Research of the Russian Academy of Science. (In Russian)

https://ozlib.com/853031/tehnika/etapy_razvitiya_mirovoy_energetiki

[15] Lovelock, J.E. and Margulis, L. (1974) Atmospheric Homeostasis by and for the Biosphere: The Gaia Hypothesis. Tellus, 26, 2-10. https://doi.org/10.3402/tellusa.v26i1-2.9731

[16] Dawkins, R. (1982). The Extended Phenotype: Long Reach of the Gene. Oxford University Press. 